\title{
Comparitive Study of Antihypertensive Treatment with Enalapril and Atenolol on Oxidative Stress and Insulin Resistance in Essential Hypertension
}

\author{
Sankar $\mathrm{P}^{1}$, Megha Ja ${ }^{1}$, Zachariah Bobby ${ }^{1}$ * \\ ${ }^{I}$ Department of Biochemistry, Jawaharlal Institute of Postgraduate Medical Education and Research \\ [JIPMER], Puducherry - 605 006, India \\ * Correspondence: Dr. Zachariah Bobby, Professor \& Head of Biochemistry, \\ JIPMER, Puducherry - 605 006, India. \\ Mobile No: + 9194436 02996, Phone: +91-413-2273078, \\ E-mail ID: sankarjipmer@gmail.com, zacbobby@yahoo.com, \\ Fax: 0413-2272067/66
}

\begin{abstract}
Hypertension is a heterogenous disorder in which patients can be stratified by pathophysiology characteristics that have a direct bearing, on the efficacy of specifically targeted antihypertensive medications, on the detection of potentially curable forms of hypertension and on the risk of cardiovascular complications. The study is done to compare the effect of antihypertensive treatment with Enalapril and Atenolol on oxidative stress and insulin resistance in patients with essential hypertension .Plasma Malonaldehyde(MDA), reduced Glutathione and Catalase were estimated to measure oxidative stress and fasting plasma Insulin to measure Insulin resistance.Total 57 hypertensive patients(31 patients on Enalapril and 26 on Atenolol drug) and 28 healthy controls are taken in the study.Plasma MDA was estimated by the method of Yagi.(Yagi 1984),erythrocyte reduced Glutathione by method of Beatle et.al..Catalase activity in erythrocytes estimated by method of Aebi (Aebi,1984).Fasting plasma insulin estimated by using human insulin ELISA kit.The data is statistically analysed and the mean, standard deviation values were calculated.In the present study, we found that systolic blood pressure $(S B P)$,diastolic blood pressure $(D B P)$,Oxidative stress and insulin resistance were higher in hypertensive patients.Treatment with Enalapril reduces $S B P, D B P$, Oxidative stress, insulin resistance and improves antioxidant system and insulin signalling more significantly than Atenolol.
\end{abstract}

Key words: Atenolol,,Catalase,Enalapril,Insulin resistance,Malonaldehyde, oxidative stress

\section{Introduction:}

Hypertension is a multi-factorial process, prevalent in developed as well as in developing countries [1]. Hypertension can result from either due to increased cardiac output or increased peripheral resistance or both. At present, essential hypertension is treatable but not curable. Effective lowering of the BP has been effected by drugs that block alpha-adrenergic receptors either in the peripheral or in the central nervous system, betablocker, angiotensin converting enzyme inhibitors and calcium channels inhibitors [2]. Compared to the normal subjects, the essential hypertensive patients have increased oxidative stress [3]. Whether oxidative stress precedes hypertension or hypertension precedes oxidative stress is still unknown.

Several in vitro studies have documented that, essential hypertensive patients are more prone to oxidative stress than normotensive subjects because of an increased reactive oxygen species (ROSs) production [4]. Most of the essential hypertensive patients showed as increased activity of rennin angiotensin system [5].which can leads to increased production of Ang II from Ang I. Ang-II activates the membrane-bound NADH and NADPH oxidases [6].The increased vascular activity of NADH and NADPH oxidase enhances the production of reactive oxygen species by several pathways, including the increased activation of xanthine oxidase, the auto-oxidation of NADH, and the inactivation of superoxide dismutase.[6].

Under physiological condition, reactive oxygen species (ROSs) are produced in the course of normal metabolism is fully inactivated by an elaborate cellular and extra cellular antioxidant defense system. Oxidative stress occurs when redox homoeostasis within the cell is altered. This imbalance may be due to either an over production of ROS or deficiency of an antioxidant system.

In mammalian cells, there are several mechanisms by which an organism defends itself against oxidative stress. Among them, there are small molecular antioxidants such as reduced glutathione (GSH) and antioxidant scavenging enzymes such as cellular $\mathrm{Cu}$, Zn super oxide dismutase (SOD-1), catalase (CAT), cellular glutathione peroxidase (cGSH-Px) and glutathione S-transferase (GST) [7]. Sato et al. [8] postulated that SOD-1 is a key enzyme in protecting the vessel wall against oxidative injury. Measurement of the activity of both glutathione-dependent enzymes may serve as an estimation of functioning of antioxidant system. GSH is a co substrate of cGSH-Px and GST, and in reactions with peroxides, it is converted to glutathione disulfide. 
GSH is responsible for protecting cellular thiol against oxidation. The levels of GSH fluctuate under various physiological conditions, including aging and hypertension, which are accompanied by increased lipid peroxidation [9]. The study of oxidant and antioxidant imbalance is therefore useful in essential hypertension in order to evaluate oxidative stress. So, any antihypertensive drugs which enhance the antioxidant system may be useful to reduce the oxidative stress in essential hypertension.

Insulin resistance and hypertension are common disorders that are closely related. Insulin resistance (IR) is defined as an impaired metabolic response to either exogenous or endogenous insulin, which results in a higher plasma insulin concentration than would be expected for the existing plasma glucose Among several factors, oxidative stress has been reported to be intimately related to these diseases [Rudenski AS et al 1999]. Several studies in vitro have demonstrated that reactive oxygen species (ROS) can cause insulin resistance by several mechanisms, such as increased serine/tyrosine phosphorylation leading to inhibition of insulin receptor substrate-1 (IRS-1) phosphorylation and inactivation of phosphatidylinositol (PI)-3 kinase activities. All these effects leads to inactivation/inhibition of other substrates involved in the insulin signaling pathway like protein kinase B (PKB), IP3, Grb2 and etc and there by causing insulin resistance [10 ]. Folli et al reported that, increased angiotensin II in essential hypertension inhibits the insulin signaling pathway in aortic smooth muscles at multiple levels such as inhibition of phosphatidylinositol (PI)-3 kinase activation associated with IRS-1[10].

Several studies reported that, treatment with ACE inhibitors improves the insulin resistances. Administration of ACE inhibitor reduces the angiotensin II formed from angiotensin I by ACE. Which in turn reduces the oxidative stress and serine/tyrosine phosphorylation which causes the inhibition of insulin receptor substrate-1 (IRS-1) phosphorylation and inactivation of phosphatidylinositol (PI)-3 kinase and there by improves insulin signalling pathway. .

\section{Materials And Methods:}

2.1. Subjects: Subjects were classified as normotensive (or control) and hypertensive as per the recommendation of the JNC 7 report [Chobanian AV et al 2003]. 57 freshly diagnosed essential 1 hypertensive patients and 28 normotensive subjects in the age group of 30-60 years were enrolled for this study. Subjects with history of diabetes, renal disease, endocrine dysfunction, coronary heart disease infections, smokers, alcoholics and those who are on any kind of medications were excluded from the study. A written informed consent was obtained from the subjects.

\subsection{Blood sample collection:}

On the day of the study, subjects were reported to our laboratory in the morning after an overnight fasting of 12 hours. Five $\mathrm{ml}$ of venous blood was collected from them in bottles containing EDTA. The whole blood will be used for estimation of glutathione and hemoglobin. Plasma was collected by centrifuging rest of the sample at $3000 \mathrm{~g}$ for $5 \mathrm{~min}$ at $4^{\mathrm{O}} \mathrm{C}$ and was used for the estimation of malondialdehyde, protein carbonylation, insulin and lipid profile. The erythrocytes separated will be used for the estimation of antioxidant enzymes and rest of the plasma will be stored at $-70^{\circ} \mathrm{C}$ until analysis.

\subsection{Anthropometric measurements:}

Height and weight were measured by using a standardized protocol, and BMI was calculated as weight in kilograms divided by square of height in meters.

\subsection{Analysis of plasma biochemical parameters:}

Plasma glucose and lipid profile were estimated in fasting samples using standard reagent kit adapted to the 550 Express Plus clinical chemistry analyzer (Bayers Diagnostics, USA). Fasting plasma insulin was estimated using human insulin ELISA kit following manufactures (United Biotech Inc, USA) instructions. From the fasting glucose and insulin values the homeostatic model assessment-insulin resistant (HOMA-IR) was calculated using the following formula (Pickavance., 2001).

(1). HOMA-IR = fasting insulin $(\mu \mathrm{U} / \mathrm{ml}) \mathrm{X}$ fasting glucose $(\mathrm{mM} / \mathrm{L}) / 22.5$.

\subsection{Analysis of oxidative stress parameters}

The plasma MDA was estimated by the method of Yagi (Yagi. 1984). To $0.5 \mathrm{ml}$ of plasma, $2.5 \mathrm{ml}$ of trichloroacetic acid (20\% TCA) was mixed and incubated at room temperature for 10 minutes. After 10 minutes the mixture was centrifuged at $3500 \mathrm{~g}$ for 10 minutes at room temperature. The supernatant was discarded and the precipitate was washed twice with $0.05 \mathrm{M} \mathrm{H}_{2} \mathrm{SO}_{4}$. The precipitate was dissolved in $2.0 \mathrm{ml}$ of $0.05 \mathrm{M} \mathrm{H}_{2} \mathrm{SO}_{4}$ and $3.0 \mathrm{ml}$ of thiobarbituric acid (TBA) reagent $\left(0.22 \% \mathrm{TBA}\right.$ in $2 \mathrm{M} \mathrm{Na}_{2} \mathrm{SO}_{4}$ solution). This mixture was incubated at $95^{\circ} \mathrm{C}$ in a boiling water bath for 1 hour. After 1 hour, the tubes were cooled under running tap water and mixed thoroughly with $4.0 \mathrm{ml}$ of butanol. The tubes were centrifuged at $3500 \mathrm{~g}$ for 15 minutes at room 
temperature. The absorbance of butanol layer was measured at $530 \mathrm{~nm}$. The concentration of MDA was calculated using the molar extinction co-efficient $\left(1.56 \times 10^{5}\right)$ and expressed as $\mu \mathrm{Mols} / \mathrm{L}$.

\subsection{Protein carbonylation}

The erythrocyte reduced glutathione content was determined by the method of Beutle et al (Beutle et al., 1963). To $0.2 \mathrm{ml}$ of whole blood, $1.8 \mathrm{ml}$ of milliQ water and $3.0 \mathrm{ml}$ of precipitating solution (5\% TCA and 1mM EDTA) was added and mixed. This mixture was incubated at room temperature for 10 minutes. After 10 minutes the mixture was centrifuged at $3500 \mathrm{~g}$ for 10 minutes. To $1.5 \mathrm{ml}$ of the supernatant, $2.5 \mathrm{ml}$ of $\mathrm{Na}_{2} \mathrm{HPO}_{4}(0.3 \mathrm{M})$ and $3.0 \mathrm{ml}$ of dithionitro benzoic acid $(0.4 \%$ DTNB and $1 \%$ sodium citrate) was added. This mixture was incubated at room temperature for 30 minutes. After 30 minutes of incubation the absorbance was measured at $412 \mathrm{~nm}$. A standard graph was drawn using reduced glutathione as standard and the reduced glutathione concentration in the samples were calculated. The hemoglobin content of the blood was estimated using Drabkin's solution following manufacture's instructions. The reduced glutathione values are expressed as $\mathrm{mg} / \mathrm{g} \mathrm{Hb}$.

The catalase activity in erythrocytes was estimated by the method of Aebi (Aebi., 1984). Red blood cells (RBC) were separated from the blood by centrifuging at $3500 \mathrm{~g}$ for 10 minutes at $4^{0} \mathrm{C}$. RBC pellet was washed three times with icecold PBS. RBC lysate was prepared by adding $0.1 \mathrm{ml}$ of $\mathrm{RBC}$ to $0.4 \mathrm{ml}$ of milliQ water. $20 \mu \mathrm{l}$ of RBC lysate was further diluted in $10 \mathrm{ml}$ of phosphate buffer $\left(50 \mathrm{mM} \mathrm{KH}_{2} \mathrm{PO}_{4}\right.$ and $\left.50 \mathrm{mMNa}_{2} \mathrm{HPO}_{4}, \mathrm{pH} 7.0\right)$. Catalase assay was performed by adding $1.0 \mathrm{ml}$ of $30 \mathrm{mM} \mathrm{H}_{2} \mathrm{O}_{2}$ to $2.0 \mathrm{ml}$ of diluted $\mathrm{RBC}$ lysate. The decomposition of $\mathrm{H}_{2} \mathrm{O}_{2}$ was measured by monitoring the decrease in the absorbance at $240 \mathrm{~nm}$ for 60 seconds. The catalase activity was expressed as rate constant $(\mathrm{k} / \mathrm{ml})$.

\subsection{Antihypertensive treatment:}

The essential hypertensive patients were followed after two months of treatment with ACE-inhibitor enalapril and beta blocker atenolol.

\section{Statistical Analysis}

Results were shown as mean \pm SD. Statistical significance of difference between control, hypertensive patients with before treatment and two months after treatment was evaluated using one way ANOVA analysis by Tukey method. Correlation between the clinical parameters was estimated by Pearson's Correlation Coefficient. A P-value less than 0.05 was considered statistically significance. All calculations were performed using the SPSS version 13.0 for windows.

IV. Results And Discussion:

Figure.1.The mean \pm SD values of various parameters of controls and cases on Enalapril and Atenolol

\begin{tabular}{|c|c|c|c|c|c|c|}
\hline S.No & Parameters & $\begin{array}{l}\text { Control } \\
(\mathrm{N}=28)\end{array}$ & \multicolumn{2}{|c|}{$\begin{array}{c}\text { Enalapril group } \\
(\mathrm{N}=31)\end{array}$} & \multicolumn{2}{|c|}{$\begin{array}{c}\text { Atenolol group } \\
(\mathrm{N}=26)\end{array}$} \\
\hline & & & Before & After & Before & After \\
\hline $\begin{array}{l}2 \\
3 \\
4 \\
5 \\
6 \\
7 \\
8 \\
9 \\
10 \\
11 \\
12 \\
13 \\
14 \\
15 \\
16 \\
17\end{array}$ & $\begin{array}{l}\text { Age (years) } \\
\text { BMI }\left(\mathrm{Kg} / \mathrm{m}^{2}\right) \\
\text { SBP }(\mathrm{mmHg}) \\
\text { DBP }(\mathrm{mmHg}) \\
\text { GSH }(\mathrm{mg} / \mathrm{g} \mathrm{Hb}) \\
\text { GPx }(\mathrm{U} / \mathrm{g} \mathrm{Hb}) \\
\text { Catalase }(\mathrm{k} / \mathrm{ml}) \\
\text { MDA ( } \mu \mathrm{mol} / \mathrm{L}) \\
\text { Protein } \\
\text { Carbonyls } \\
\text { (nmol/mg) } \\
\text { Glucose (mg/dL) } \\
\text { Insulin ( } \mu \mathrm{d} / \mathrm{mL}) \\
\text { HOMA-IR } \\
\text { Cholesterol } \\
\text { (mg/dL) } \\
\text { TG (mg/dL) } \\
\text { HDL (mg/dL) } \\
\text { LDL (mg/dL) } \\
\text { VLDL (mg/dL) }\end{array}$ & $\begin{aligned} 39.1 & \pm 6.4 \\
23.7 & \pm 2.6 \\
105.1 & \pm 12.8 \\
71.1 & \pm 7.9 \\
2.8 & \pm 0.6 \\
57.8 & \pm 18.8 \\
32.3 & \pm 8.8 \\
2.5 & \pm 0.9 \\
2.2 & \pm 0.8 \\
81.6 & \pm 11.8 \\
24.7 & \pm 10.8 \\
4.9 & \pm 2.3 \\
168.4 & \pm 34.3 \\
127.7 & \pm 88.9 \\
49.7 & \pm 9.7 \\
94 & \pm 34.7 \\
25.4 & \pm 17.7\end{aligned}$ & $\begin{array}{c}38.4 \pm 5 \\
25.6 \pm 3.1 \\
165.9 \pm 19.8 \\
99.8 \pm 5.7 \\
1.8 \pm 0.5 \\
90.6 \pm 10.5 \\
27 \pm 3.4 \\
4.9 \pm 0.9 \\
5.5 \pm 1 \\
97.6 \pm 4.9 \\
49.1 \pm 34.6 \\
12.6 \pm 8.9 \\
195 \pm 40.9 \\
136 \pm 38.9 \\
36.9 \pm 3.7 \\
125.7 \pm 36.1 \\
27.5 \pm 7.1\end{array}$ & $\begin{array}{c}38.4 \pm 5 \\
25.5 \pm 3.1 \\
115.5 \pm \\
11.2 \\
72.4 \pm 6.5 \\
2.7 \pm 0.8 \\
74.1 \pm 9.1 \\
33.4 \pm 3.7 \\
2.3 \pm 0.7 \\
2.4 \pm 0.9 \\
85.2 \pm 9.8 \\
22.5 \pm \\
17.1 \\
4.6 \pm 3.3 \\
175.7 \pm \\
22.2 \\
122.6 \pm \\
32.4 \\
42.2 \pm 4.9 \\
100.9 \pm \\
22.9 \\
24.5 \pm 6.5\end{array}$ & $\begin{array}{c}38.6 \pm 5.5 \\
25.3 \pm 2.5 \\
167.7 \pm 18.1 \\
100.2 \pm 5.4 \\
2.2 \pm 0.5 \\
88.8 \pm 9 \\
27.5 \pm 3.7 \\
5 \pm 1.2 \\
5.5 \pm 1 \\
90.4 \pm 14.1 \\
36.3 \pm 30.9 \\
8 \pm 6.9 \\
190.4 \pm 37.3 \\
137.6 \pm 46.8 \\
39.1 \pm 6.6 \\
122.1 \pm 36.5 \\
27.7 \pm 8.9\end{array}$ & $\begin{array}{c}38.6 \pm 5.5 \\
25.2 \pm 2.5 \\
127.3 \pm \\
6.8 \\
78.6 \pm 4.8 \\
1.4 \pm 0.4 \\
86 \pm 7.6 \\
26.1 \pm 2.8 \\
3.8 \pm 0.9 \\
3.6 \pm 0.7 \\
94.5 \pm 9.5 \\
47.9 \pm \\
32.7 \\
11.1 \pm 7.8 \\
196.9 \pm \\
30.7 \\
142.8 \pm \\
55.5 \\
38.9 \pm 4.7 \\
133.6 \pm 28 \\
27.9 \pm \\
11.1\end{array}$ \\
\hline
\end{tabular}

Most of the essential hypertensive patients showed as increased activity of rennin angiotensin system [5].which can leads to increased production of Ang II from Ang I. Ang-II activates the membrane-bound NADH and NADPH oxidases [6].The increased vascular activity of NADH and NADPH oxidase enhances the production 
of reactive oxygen species by several pathways, including the increased activation of xanthine oxidase, the autooxidation of $\mathrm{NADH}$, and the inactivation of superoxide dismutase [6].

Insulin resistance and hypertension are common disorders that are closely related. Among several factors, oxidative stress has been reported to be intimately related to these diseases [Rudenski AS et al 1999]. Several studies in vitro have demonstrated that reactive oxygen species (ROS) can cause insulin resistance by several mechanisms, such as increased serine/tyrosine phosphorylation leading to inhibition of insulin receptor substrate-1 (IRS-1) phosphorylation and inactivation of phosphatidylinositol (PI)-3 kinase activities. All these effects leads to inactivation/inhibition of other substrates involved in the insulin signaling pathway like protein kinase B (PKB), IP3, Grb2 and etc and there by causing insulin resistance [10 ]. Folli et al reported that, increased angiotensin II in essential hypertension inhibits the insulin signaling pathway in aortic smooth muscles at multiple levels such as inhibition of phosphatidylinositol (PI)-3 kinase activation associated with IRS-1[10].

Several studies reported that, treatment with ACE inhibitors improves the insulin resistances. Administration of ACE inhibitor reduces the angiotensin II formed from angiotensin I by ACE. Which in turn reduces the oxidative stress and serine/tyrosine phosphorylation which causes the inhibition of insulin receptor substrate-1 (IRS-1) phosphorylation and inactivation of phosphatidylinositol (PI)-3 kinase activities and there by improves the insulin signaling pathway [Haffner, S. M. 2000].

In this present study, we found that, the SBP, DBP, plasma malondialdehyde (MDA), protein carbonyl contents, RBC glutathione peroxidase (GSH-Px), glucose, insulin and HOMA-IR were significantly higher in essential hypertensive patients as compare to control subjects; whereas whole blood glutathione (GSH), RBC catalase were significantly lower in essential hypertensive patients. But, there is no significant difference in BMI between control subjects and essential hypertensive patients.

After enalapril treatment, the systolic BP, diastolic BP, glutathione peroxidase, plasma malondialdehyde (MDA), protein carbonyl contents, glucose, insulin, HOMA-IR and LDL were significantly reduced as compared to before treatment. Whereas the whole blood glutathione (GSH), catalase and HDL were significantly increased. But there are no significant changes in cholesterol, TG, VLDL.

After atenolol treatment, the systolic BP, diastolic BP, whole blood glutathione (GSH), plasma malondialdehyde (MDA), protein carbonyl contents, were significantly reduced as compared to before treatment. Whereas HDL was significantly increased. But there are no significant changes in catalase, glutathione peroxidase, insulin, HOMA-IR and lipid profile.

In that present study, we also observed that, the enalapril treatment reduces SBP, DBP, GSH-Px, protein carbonyl contents, malondialdehyde, glucose, insulin, HOMA-IR, LDL and increases the GSH, catalase and HDL significantly than atenolol treatment.

\section{Reference:}

[1] Kashyap MK, Yadav V, Sherawat BS, Jain S, Kumari S, Khullar M, Sharma PC, Nath R. Different antioxidants status, total antioxidant power and free radicals in essential hypertension. Mol Cell Biochem. 2005 Sep; 277 (1-2):89-99.

[2] William F.Ganong. Review of Medical physiology.19 $9^{\text {th }}$ edition; 613: LANGE publication.

[3] Ward NC,Croft KD. Hypertension and oxidative stress. Clin Exp Pharmacol Physiol.2006; 33(9); 872-876.

[4] Digiesi V, Oliviero C, Giannò V, Rossetti M, Fiorillo C, Oradei A, Lenuzza M, Nassi P. Reactive metabolites of oxygen, lipid peroxidation, total antioxidant capacity and vitamin $\mathrm{E}$ in essential arterial hypertension. Clin Ter. 1997 Nov; 148(11):515-9.

[5] Folli F,Khan CR et al.Angiotensin II inhibits the Insulin signalling in aortic smooth muscles at multiple levels:a potential role of Serine Phosphorylation in Insulin/Angiotensin crosstalk .J .Clin Invest. 1997;100:2158-2169.

[6] Mathews DR, Hosker JP, Rudenski AS,Naylor BA,Treacher DF,Turner RC 1985.Homeostasis model assessment: insulin resistance and cell function from fasting plasma glucose and insulin concentration in man. (Diabetologia 28 )

[7] Kasapoglu, M. and Ozben, T. Alterations of antioxidant enzymes and oxidative stress markers in aging. Exp. Gerontol. 36 (2001) 209220.

[8] Sato, M., Yanagisawa, H., Nojima, Y., Tamura, J. and Wada, O. Zn deficiency aggravates hypertension in spontaneously hypertensive rats: possible role of $\mathrm{Cu} / \mathrm{Zn}$-superoxide dismutase. Clin. Exp. Hypertension. 24(2002) 355-370.

[9] Zhou, X.J., Vaziri, N.D., Wang, X.Q., Silva, F.G. and Laszik, Z. Nitric oxide synthase expression in hypertension induced by inhibition of glutathione synthase. J. Pharmacol. Exp. Therap. 300 (2002) 762-767.

[10] Haffner SM(2000) Clinical relevance of the oxidative concept. (Metabolism )49;30-34. 